\title{
Lidil
}

Revue de linguistique et de didactique des langues

$52 \mid 2015$

Les pratiques artistiques dans l'apprentissage des langues

\section{Exercice d'écriture sous contrainte et engagement du lecteur-scripteur de FLE}

A Constrained Writing Task and the Engagement of the Reader/Writer of French as a Foreign Language

\section{Christèle Maizonniaux}

\section{OpenEdition \\ Journals}

Édition électronique

URL : http://journals.openedition.org/lidil/3883

DOI : $10.4000 /$ lidil.3883

ISSN : 1960-6052

\section{Éditeur}

UGA Éditions/Université Grenoble Alpes

\section{Édition imprimée}

Date de publication : 20 novembre 2015

Pagination : 177-197

ISBN : 978-2-84310-312-4

ISSN : $1146-6480$

Référence électronique

Christèle Maizonniaux, «Exercice d'écriture sous contrainte et engagement du lecteur-scripteur de FLE », Lidil [En ligne], 52 | 2015, mis en ligne le 01 janvier 2017, consulté le 30 octobre 2020. URL http://journals.openedition.org/lidil/3883 ; DOI : https://doi.org/10.4000/lidil.3883 


\title{
Exercice d'écriture sous contrainte et engagement du lecteur-scripteur de FLE
}

\author{
Christèle Maizonniaux*
}

\begin{abstract}
RÉSUMÉ
Si des recherches ont été effectuées en français langue maternelle concernant l'engagement du lecteur-scripteur dans le cadre d'exercices d'écriture sous contrainte, force est de constater, en revanche, que dans le champ du français langue étrangère, peu de travaux ont à ce jour été conduits. Nous nous intéresserons ici aux traces d'engagement présentes dans un exercice d'écriture sous contrainte de type «greffe», réalisé par des apprenants adultes de français de niveau A1-A2 du CECR, à la suite de la lecture du livre Pochée de Florence Seyvos, un ouvrage publié dans une collection pour la jeunesse. La recherche a été conduite en contexte universitaire à l'Australian National University (Canberra) auprès de 26 apprenants de français. Les résultats de l'analyse mettent à jour un foisonnement de traces d'engagement et révèlent la pertinence d'exercices d'écriture ambitieux à un niveau peu élevé de maitrise de la langue cible.
\end{abstract}

\begin{abstract}
While research has been undertaken in the field of French as a first language concerning the extent of the reader/writer's engagement in constrained writing exercises, it is notable that in the field of French as a foreign language, however, very little work has as yet been undertaken. In this article, we analyse the signs of engagement that are apparent in the product of a constrained writing exercise that involved the insertion of a new event into the existing storyline, written by adult students of French at CECR level A1-A2 in response to reading the book Pochée by Florence Seyvos, a work published for young readers. The research was undertaken in a university context at The Australian National University (Canberra) and involved 26 students of French. The results of the analysis reveal an abundant tracery of involvement, and also show
\end{abstract}

* Flinders University (Australie) et Litextra, Université Grenoble Alpes. 
the relevance of ambitious writing exercises at a relatively low level of mastery of the target language.

\section{Introduction}

Dans l'ouvrage Cours de didactique du français langue étrangère et seconde paru en 2005, Cuq et Gruca (2005) font remarquer «qu'il est encore trop rare dans les méthodes qu'on fasse écrire les apprenants dans le but de développer une véritable compétence textuelle» (p. 184). De fait, une analyse des grilles concernant l'écrit du Cadre européen commun de référence pour les langues (CECR, Conseil de l'Europe, 2001), à partir duquel sont conçus les manuels de langue en Europe, montre que cet outil cantonne l'apprenant, pour les niveaux qui nous concernent (A1-A2), à l'écriture de «bribes de texte», de fragments. Dans cet outil de référence, la notion de «texte», n'apparait qu'au niveau B1, c'est-à-dire au niveau «utilisateur indépendant». En outre, les exercices se rattachant à l'écriture littéraire n'apparaissent qu'en fin de parcours dans la grille d'auto-évaluation proposée par le CECR.

Pour autant, le domaine de l'écriture créative en langue étrangère n'est pas inexistant. Il s'est développé sur la base de pratiques déjà éprouvées dans les ateliers d'écriture, en particulier en ce qui concerne les activités basées sur le couple déclencheur/écrit court ${ }^{1}$, et fait toujours l'objet d'un certain intérêt, comme en témoigne la parution récente aux Presses universitaires de Grenoble d'un ouvrage intitulé Écritures créatives (Rodier, Bara \& Bonvallet, 2011).

Pour ce qui est des écrits créatifs jouant sur le couple modèle de texte/production de texte ${ }^{2}$, ce domaine est encore peu exploré en langue étrangère. En revanche, il fait l'objet de recherches importantes dans le champ du français langue maternelle. On pense ici à l'écriture d'invention mise en place au lycée (on pourra se référer ici, entre autres, à Huyn [2004] ou Le Goff [2008]). On pense aussi à d'autres recherches conduites en français langue maternelle en contexte universitaire et basées sur le principe des ateliers d'écriture, avec, par exemple, un travail autour de l'intertextualité (Houdart-Mérot, 2008).

1. Il s'agit par exemple de créer un poème personnel à partir d'un canevas très simple (ce peut être un extrait de poème) fourni par l'enseignant. C'est ce canevas qui sert de déclencheur à l'écriture.

2. On entend par là à la fois les écrits jouant sur l'imitation de modèles et ceux jouant sur la production à partir d'un modèle. 
L'objectif de notre recherche est d'analyser la pertinence d'exercices d'écriture créative en lien avec la lecture de récits illustrés, destinés à la jeunesse pour l'enseignement-apprentissage du français langue étrangère, en contexte universitaire et à un niveau peu élevé de maitrise de la langue cible. À partir de productions d'apprenants de type «greffe», nous voulons déterminer, ce qui dans ces productions témoigne de l'investissement de l'apprenant au cours de la démarche proposée et dans la tâche d'écriture elle-même. Il s'agit également de saisir in fine, ce qui, dans un texte destiné à la jeunesse, stimule et engage de jeunes adultes.

Nous présenterons ici tout d'abord le contexte de la recherche ainsi que la conception de la lecture-écriture qui sous-tend la démarche. Nous donnerons ensuite des précisions concernant le cadre d'analyse élaboré spécifiquement pour saisir les traces d'engagement du lecteur-scripteur dans sa production et ce, après avoir présenté l'ouvrage sur lequel s'est basé ce travail d'écriture ainsi que la consigne donnée et la démarche entreprise. Nous proposerons enfin, à partir d'exemples tirés du corpus de productions, plusieurs exemples de traces d'engagement relevées.

\section{Contexte de la recherche et conception sous-tendant la démarche}

\subsection{Contexte de la recherche}

La recherche qui s'est déroulée au premier semestre 2009 a été conduite auprès de 26 apprenants de français, à l'Australian National University (Canberra). Cette université n'accueille que des étudiants ayant obtenu de très bons résultats au diplôme sanctionnant la fin des études secondaires. Les participants à la recherche font partie d'une cohorte de 80 étudiants dont la moitié environ est issue du secondaire et l'autre moitié des cours de débutants proposés dans cette institution l'année précédente. Ces derniers ont derrière eux 130 heures de français. On estime généralement que tous les apprenants ont atteint le niveau A1 du CECR et atteindront au cours ou à la fin du semestre qui nous intéresse le niveau A2 du CECR. Sur les 26 participants à l'étude, 5 mentionnent comme langue parlée à la maison une langue autre que l'anglais et deux de ces 5 apprenants sont des apprenants internationaux de nationalité chinoise. 11 étudiants du groupe sont inscrits en «Bachelor of Arts», une licence pluridisciplinaire. 9 sont inscrits en sciences, en commerce ou en langue et 6 préparent un double diplôme. 


\subsection{Une démarche qui lie étroitement lecture et écriture}

Nous avons fait le choix de proposer aux apprenants des exercices d'écriture créative jouant sur le couple modèle de texte/production de texte que nous pourrions encore définir comme «écriture sous contrainte, d'appropriation et de détournements des textes» (Houdart-Mérot, 2008, p. 125). Ce type d'activité apparait dans Le dictionnaire de didactique $d u$ français (Cuq, 2003) sous la rubrique «exercice» puisqu'on y parle «d'exercices qui visent soit la réécriture, soit l'écriture : la réduction d'un texte $[\ldots]$ ou son amplification $[\ldots]$, la transformation de texte ${ }^{3}$ [...], la réparation de texte [...] ou encore les matrices de textes ${ }^{4}{ }$ » (p. 95). C'est dans la catégorie «réparation de texte» que se range l'exercice que nous proposons ici aux apprenants puisqu'il s'agit d'une greffe. Il s'agit d'insérer un nouveau passage dans la trame narrative existante, en obéissant aux contraintes de la cohérence textuelle.

L'originalité du travail repose sur le fait que les ouvrages proposés aux apprenants sont soit des albums destinés à la jeunesse, soit des récits illustrés. Le livre retenu ici est un récit illustré pour enfants. L'objectif didactique poursuivi est de «percevoir la dimension fondamentalement oblique de la littérature : on parle de soi en empruntant la langue et les mots des autres, que l'on tente de s'approprier» (HoudartMérot, 2008, p. 137). L'écriture sous contrainte doit encore permettre «de libérer la lecture en même temps qu'elle libère l'écriture» (ibid.).

L'objectif de la présente recherche est de saisir les traces d'engagement présentes dans les productions. Pour notre part, nous postulons que l'engagement de l'apprenant dans la démarche proposée est manifeste lorsque sont présentes dans sa production les traces d'un apprentissage sur les plans linguistiques, littéraires ou culturels ou lorsque

3. La transformation de texte: «changement de genre (passage de prises de notes à un texte rédigé; transformer un récit en dialogue ou en scénario, et inversement); changement de point de vue (changement de modalité, appréciative, dépréciative, neutre, d'un commentaire; adoption du point de vue d'un autre personnage du récit; transformation d'une thèse dans un texte argumentatif)» (Cuq, 2003, p. 95).

4. Les matrices de textes : «canevas (déroulement du texte par exemple un récit); guidage par des images ou des bruits; imposition de contraintes comme la longueur, une liste de mots à utiliser, l'usage d'une structure de base, le pastiche, l'amorce à poursuivre, l'utilisation de tournures syntaxiques ou sémantiques précises » (ibid., p. 95). 
sont présents les indices de la construction d'une relation esthétique au texte littéraire étudié. On peut dire qu'on tente ici d'appréhender ce qui reste d'une séquence didactique ${ }^{5}$. On peut observer en quoi ce type d'exercice suscite le réemploi tel quel du texte source et aussi repérer ce qui est de l'ordre de la reformulation personnelle. Une de nos hypothèses est qu' 'engagement» et qualité du récit sont liés. Par ailleurs, nous voulons également saisir des indicateurs concernant l'investissement de l'apprenant lecteur-scripteur de langue étrangère dans la tâche d'écriture elle-même.

La notion d' «investissement de l'écriture» définie par Barré-De Miniac $(2000$, 2002) dans le cadre de l'enseignement-apprentissage du français langue maternelle est intéressante ici. L'investissement de l'écriture est, selon Barré-De Miniac, une des quatre dimensions du rapport à l'écriture. Il peut être défini comme

[...] l'intérêt affectif pour l'écriture et de la quantité d'énergie que l'on y consacre [...]. Mais deux aspects doivent soigneusement être distingués : la force de l'investissement et le type d'investissement. La force désigne l'intensité de l'investissement [...]. Le type d'investissement, lui, se réfère aux objets, c'est-à-dire aux situations d'écriture et aux types de textes sur lesquels se porte l'investissement plus ou moins fort des scripteurs. (Barré-De Miniac, 2002, paragr. 11-12)

On peut dire que ces deux aspects sont également pertinents dans le cadre de l'enseignement-apprentissage du français langue étrangère. Ils sont cependant, de notre point de vue, insuffisants pour appréhender la complexité de la lecture-écriture en langue étrangère. Retenir la notion d'«engagement» permet de mieux tenir compte des spécificités de la lecture-écriture en langue étrangère et de la variété des indices relevés.

5. Signalons ici que Daunay a publié en 2004 un article intitulé : «Traces d'apprentissages : que reste-t-il d'une séquence didactique?» Il y analyse à la fois un corpus de travaux d'élèves et sur un corpus des discours des élèves par rapport à l'objet d'apprentissage (le discours indirect libre). 


\section{Présentation de l'ouvrage retenu, de l'exercice proposé et du cadre d'analyse}

\subsection{Pochée, ou comment vivre après la mort de l'être aimé}

Pochée est l'histoire d'une jeune tortue qui, partie de chez elle pour «vivre comme une grande», a rencontré l'âme sœur. Hélas, un jour Pouce, son compagnon, meurt accidentellement. Pochée passe ensuite quelques jours dans sa cabane et reçoit la visite de ses parents et de Truc, un petit escargot. Elle décide alors de partir en voyage. Elle rencontre Nestor, une autre tortue qui lui fait les yeux doux mais qu'elle n'aime pas et Pépin, un hérisson qui devient son ami. Après une ellipse, on retrouve Pochée âgée de 101 ans entourée par ses 19 petits-enfants, uniquement des filles.

\subsection{Les principales caractéristiques de cet ouvrage}

Cet ouvrage mêle le texte de Florence Seyvos, un auteur reconnu de littérature générale qui a, entre autres, obtenu le prix Goncourt du premier roman en 1995 et les images de Claude Ponti, un des grands auteurs contemporains d'albums pour enfants. Il présente plusieurs caractéristiques d'accessibilité : sa longueur (63 pages), l'alternance texte-image, sa grande lisibilité visuelle et son texte aéré, la présence de marques d'attention à l'adresse d'un lecteur novice, l'image qui illustre et donne à voir ce que le texte dit. On peut encore dire que le style de Seyvos qualifié par certains de «minimaliste» (Nières-Chevrel, 2009, p. 85), est accessible car il se caractérise par des phrases courtes, une syntaxe peu compliquée. Sur le fond, il emprunte à deux genres bien connus : le conte et la fable. Ce livre est également un récit linéaire, le récit d'une quête. On y retrouve la thématique bien connue du voyage initiatique. Ces différentes caractéristiques sont essentielles pour un public d'apprenants qui ne maitrise pas encore bien la langue cible. Néanmoins, proposer des ouvrages de littérature de jeunesse à un public d'adultes matures et exigeants ne va pas de soi. Il y a là un vrai paradoxe. Les traits de complexité présents dans Pochée ont une importance cruciale, non seulement pour susciter l'intérêt des apprenants dans la lecture et la découverte du livre, mais aussi pour envisager une démarche pédagogique pouvant déboucher sur des écrits fructueux. Pochée, en effet, est un ouvrage à portée philosophique et psychologique qui aborde les thèmes de la mort et du deuil, mais aussi de l'amitié et de la destinée. Sa complexité se niche également dans un procédé d'écriture où sont insérées dans le récit linéaire des lettres que le personnage principal adresse 
à son défunt compagnon ainsi que des lettres fictives que Pochée s'écrit en retour à elle-même. On pourrait encore citer parmi les éléments concourant à la complexité de ce texte sa dimension culturelle et la référence à la fable, l'importance de la symbolique, l'emploi des temps ou encore l'intericonicité ${ }^{6}$, qui est à l'image ce que l'intertextualité est au texte.

\subsection{L'exercice d'écriture sous contrainte proposé}

L'exercice d'écriture proposé s'est déroulé sur une séance d'une heure en fin de parcours. Les apprenants ne disposaient pas de l'ouvrage pendant cette séance. Les productions analysées constituent le premier jet et s'inscrivent dans la phase finale d'évaluation du semestre de cours. Les contraintes institutionnelles n'ont pas permis que soient collectées et analysées des réécritures de ces productions. Pour ce qui est des activités collectives organisées en classe, elles ont été basées sur des fiches de lecture à remplir à la maison et sur des activités orales de rappel. Ont été également incluses dans la démarche des activités d'écriture créative sous contrainte ${ }^{7}$, réalisées à la maison et soumises au professeur par voie électronique. Notons que seules deux séances ont été consacrées en classe à l'ouvrage retenu. Par conséquent, la plus grande partie du travail réalisé par les apprenants s'est effectuée en dehors du temps de classe. La consigne était la suivante :

Pochée vit maintenant dans sa nouvelle maison (la grotte). Elle rencontre Oscar le castor. Elle trouve Oscar très sympathique. Pochée commence à parler à Oscar de sa vie mais les parents d'Oscar arrivent. Alors Pochée s'en va et décide d'écrire une lettre à Oscar. Dans sa lettre Pochée parle de ses rencontres et de ses sentiments. Elle parle :

$1:$ de Truc, l'escargot (passage étudié en classe);

$2:$ au choix, de Nestor la tortue ou de Pépin le hérisson.

You might want to refer to other parts of the book as well. $(200 \text { words minimum })^{8}$.

6. Pour mieux saisir quelques-uns des traits de l'intericonicité dans la littérature de jeunesse contemporaine on pourra se référer, entre autres, à NièresChevrel (2008).

7. Il s'agit ici de l'écriture de lettres (écrites par le personnage principal ou par des personnages secondaires). Elles sont, elles aussi, reliées à la trame narrative.

8. Pour inviter les apprenants à faire référence, s'ils le souhaitent, à d'autres passages du livre et pour être sûre que cette possibilité pouvait être exploitée, 
L'apprenant-scripteur est ici investi d'une double mission qui consiste à effectuer un rappel de certaines parties du texte tout en donnant à voir les pensées et sentiments du personnage. Comment l'apprenant de FLE s'est-il acquitté de ces deux missions? Ce livre, si singulier, a-t-il stimulé l'expression de sentiments et l'analyse psychologique de la part de lecteurs-scripteurs adultes?

\subsection{Le cadre d'analyse mis en place}

Le cadre d'analyse devait permettre de saisir l'engagement de l'apprenant, dans la lecture du texte étudié, dans l'écriture et plus largement dans son apprentissage, à travers notamment la mémorisation du lexique, des structures grammaticales et du contenu culturel éventuel.

Le cadre que nous avons élaboré est centré sur les 4 grandes notions que sont (1) la créativité, entendue au sens de "place de l'imaginaire dans la production», (2) la créativité dans le domaine linguistique, (3) la «construction d'un rapport intime à l'écriture littéraire » (Demougin, 1998) et (4) le texte du lecteur.

\subsubsection{La créativité entendue au sens de "place de l'imaginaire dans la production"}

De manière concrète, ce critère nous permet de différencier, dans les productions réalisées après la lecture de Pochée, deux types de productions :

- celles qui sont caractérisées par une grande fidélité au texte source et où la partie «rappel» est particulièrement développée. Les sentiments des personnages y sont livrés de manière succincte;

- celles qui s'éloignent de manière plus ou moins prononcée du livre, que ce soit pour développer la partie «rappel» de manière plus personnelle, ou pour développer l'analyse des sentiments de Pochée, ou encore pour intégrer des éléments absents du texte source mais en cohérence avec celui-ci.

nous avons pris soin de mentionner cette information en anglais. Nous avons également précisé en anglais la longueur minimale requise. 


\subsubsection{La créativité dans le domaine linguistique}

Ce critère permet de saisir les traces d'appropriation linguistique (vocabulaire, grammaire) et de mettre en avant le travail de tissage opéré par l'apprenant-scripteur. En ce qui concerne la saisie de l'appropriation lexicale, les critères repris au CECR d'étendue et de maitrise sont opératoires.

Il nous faut aussi regarder comment, du point de vue linguistique, est assurée la continuité et la cohérence avec le texte source. On pourra observer si l'apprenant-scripteur cherche à élargir le champ lexical en l'enrichissant, s'il reprend le lexique du livre et comment celui-ci est repris, remodelé, détourné.

Nous considérons également que la longueur des productions constitue un indicateur d'engagement et observerons s'il y a corrélation ou non entre le degré de maitrise et la longueur de la production.

\subsubsection{La « construction d'un rapport intime avec l'écriture littéraire»}

Demougin (1998) utilise l'expression «construction d'un rapport intime avec l'écriture littéraire» pour désigner un processus à mettre en place par l'enseignant et qui consiste à proposer à l'apprenant une démarche de lecture autonome, individuelle et pertinente avec des orientations et des repères, tout en tenant compte des spécificités de l'apprenant, pour mettre ce dernier en confiance et l'amener sur le terrain de l'écriture. Nous reprenons cette expression en nous plaçant non plus du côté de l'enseignant mais du côté de l'apprenant. «La construction d'un rapport intime avec l'écriture littéraire» serait donc le résultat de la mise en place d'une démarche axée à la fois sur l'écriture et sur la lecture.

Nous empruntons à Ferrier (2006) cette définition de la littérarité : «Il y a littérarité $[\ldots]$ dès lors qu'un procédé de type littéraire $[\ldots]$ est attesté textuellement comme existant et faisant sens.» (p. 264) Si on peut objecter à cette définition qu'on ne peut conclure à la littérarité d'un article de journal simplement parce que son auteur y use d'un procédé littéraire, elle nous semble tout de même pertinente pour notre premier travail d'analyse.

On conçoit que le passage entre la création d'un rapport intime à l'écriture littéraire et l'appropriation du littéraire ancré dans la littérarité est loin d'être évident; on peut cependant imaginer que la prise de conscience de la littérarité des textes lus et surtout le repérage des traits littéraires en contexte ont pu donner lieu, par la suite, au réemploi 
de quelques traits littéraires ${ }^{9}$, témoignant ainsi d'une appropriation du littéraire, aussi modeste soit-elle.

\subsubsection{Le "texte du lecteur»}

La notion de «texte du lecteur», développée dans le champ du français langue maternelle (Rouxel \& Langlade, 2004 ; Mazauric, Fourtanier \& Langlade, 2011), place l'expérience de l'individu lecteur au centre de l'expérience littéraire et accorde une place importante à son rapport intime avec le livre. Pour nous, cette notion permet d'avoir accès à la lecture que fait le jeune adulte de Pochée, et à son interprétation personnelle. Dans l'analyse de l'ensemble des productions du corpus elle invite, par ailleurs, à établir des regroupements. Les productions sont donc ici non seulement regroupées en fonction de la fidélité du texte produit au texte source mais également en fonction du «texte du lecteur-scripteur» tel qu'il transparait dans la production, selon par exemple que le lecteur-scripteur interprète Pochée comme un conte philosophique sur l'amitié ou sur l'amour. En croisant ces informations avec les réponses fournies dans les questionnaires, ce critère permet également de saisir in fine, ce qui, dans un texte destiné à la jeunesse, stimule et engage de jeunes adultes. Il peut s'agir ici de l'humour, de l'affectif ou encore de l'émotionnel, que nous considérons, lorsqu'ils sont présents dans le texte de l'apprenant et font ainsi écho au texte littéraire, comme des indices de l'engagement du lecteur-scripteur. Ce qui pourrait alors être considéré comme la marque d'une bonne compréhension de l'exercice proposé ${ }^{10}$ marque aussi de notre point de vue, lorsque ces indices sont récurrents, que s'est établie une relation esthétique au texte littéraire et qu'il y a eu sans doute, à fortiori, un réel plaisir dans l'écriture.

\section{Vue d'ensemble du corpus de productions et traces d'engagement relevées}

\subsection{Longueur des productions et degré de maitrise}

La consigne indiquait qu'il fallait produire un texte d'au moins 200 mots. Sur les 26 productions du corpus, 2 comptent moins de 200 mots,

9. On peut penser ici, par exemple, à l'influence du style minimaliste de l'auteur.

10. Un exercice de type «greffe» requiert de la part de l'apprenant de s'assurer qu'il y a continuité et cohérence avec le texte étudié. 
8 comptent entre 200 et 250 mots, 4 comptent entre 250 et 300 mots, 6 comptent entre 300 et 400 mots et enfin, 4 sont au-delà de 400 mots, soit plus du double de ce qui était demandé.

La longueur moyenne des productions est de 293 mots. Il est à noter qu'il n'y a pas de corrélation entre la longueur des productions et la maitrise de la langue. La longueur moyenne des productions de niveau 1-2 (niveau de maitrise le plus faible) est de 311 mots. Celle des productions de niveau très élevé (niveau 4) est de 326 mots.

Nous avons ici utilisé une échelle de 1 à 4 pour indiquer le niveau linguistique des productions. Sur les 26 copies, 10 sont de niveau 4 (très bon), 4 sont de niveau 3 . Autrement dit, on peut considérer que pour 14 apprenants, soit plus de la moitié des participants à notre recherche, la langue ne pose pas problème. 8 copies sont estimées de niveau moyen et enfin 4 sont de niveau faible. Ces copies sont autant le fait d'apprenants provenant du secondaire que celles d'apprenants ayant commencé leur apprentissage du français dans notre institution.

\subsection{Les traces d'engagement relevées}

\subsubsection{Remarques préalables}

Nous rendrons compte ici des traces relevées dans quelques copies représentatives du corpus ${ }^{11}$. Les deux axes que la consigne invite à développer dans ce travail sont le rappel de deux épisodes du livre ainsi que «l'expression des sentiments» du personnage principal. Dans leurs productions, les apprenants tendent à développer un axe plus que l'autre. Ceux qui développent davantage la partie «rappel» tendent à produire des textes proches du texte source, alors que ceux qui expriment de manière plus développée les sentiments du personnage tendent à s'éloigner du texte d'appui, en intégrant, par exemple, des éléments absents du texte mais qui sont en cohérence avec celui-ci. Les productions de cette catégorie sont caractérisées par la présence lisible de l'interprétation du lecteur.

\subsubsection{Traces d'engagement relevées dans des productions proches du texte-source}

Dans ce type de production, l'impression de «collage» d'expressions prises au livre est forte. L'imaginaire de l'étudiant est pour ainsi

11. Pour un compte rendu plus complet de l'analyse des travaux réalisés, se référer à Maizonniaux (2013). 
dire absent, ou du moins, il se moule dans des formes d'expression du texte source. La production d'A8 (fig. 1) en est une illustration. Cette production, qui compte 339 mots et qui est d'un niveau linguistique satisfaisant (niveau 3), est marquée par une grande précision tant dans l'expression que dans la correction linguistique et présente une abondance de détails. On peut toutefois noter que des apprenants de niveau plus modeste proposent aussi des textes qui restent proches du texte source, mais qui, bien évidemment, comportent davantage d'erreurs. En témoigne une production plus courte, celle d'A14 (fig. 2). Pour autant et malgré sa relative brièveté ( 234 mots), ce texte recèle des traces d'engagement que nous analysons ci-dessous.

\begin{tabular}{|c|c|}
\hline $\begin{array}{c}\text { A8-PR2 } \\
\text { Sont soulignées les expressions reprises textuellement } \\
\text { du texte source. Apparaissent en gras les phrases } \\
\text { exprimant les sentiments du personnage* }\end{array}$ & $\begin{array}{l}\text { Éléments correspondants } \\
\text { du texte source (Pochée) }\end{array}$ \\
\hline $\begin{array}{l}\text { Salut Oscar, } \\
\text { Je suis heureux que je t'ai connue et je te trouve très } \\
\text { sympa! Alors je vais t'écrire de ma vie. } \\
\text { Il y a quelques mois, je suis parti de chez mes parents, } \\
\text { pour vivre comme une grande. Juste après mon départ, } \\
\text { j'ai rencontré une autre tortue qui partit de chez ses } \\
\text { parents comme moi. Elle s'appelait Pouce. ( } \rightarrow \text { p. 7) } \\
\text { Nous avons décidé de construire une cabane ensemble. } \\
\text { Ça fit facile, parce que nous étions amis immédiate- } \\
\text { ment, et étions d'accord sur tous. Notre cabane avait } \\
\text { une piscine et avait vue sur un grand lac. C'était très } \\
\text { bien. Mais un jour, un pierre tomba sur la tête de Pouce } \\
\text { et l'a tua. Je suis devenue très triste. Je voulais être } \\
\text { morte. ( } \rightarrow \text { p. 10) (Expression des sentiments) J'ai plu } \\
\text { [pleuré] longtemps. Il a plu, et le lac grossit et déborda, } \\
\text { mouillant ma cabane. L'inondation a emporté le corps } \\
\text { de Pouce. J'étais tellement triste. } \\
\text { [...] } \\
\text { J'ai lui dit au revoir, et alors j'ai trouvé cette grotte } \\
\text { magnifique. J'espère que tes parents me permettent } \\
\text { de vivre ici. Je suis gentille et jolie. (Apport person- } \\
\text { nel) Pouce me manque toujours, il m'a enseigné d'être } \\
\text { contente avec ma vie et d'être toujours positive. } \\
\text { Signé, } \\
\text { Pochée. }\end{array}$ & $\begin{array}{l}\text { «Elle était partie très tôt de chez } \\
\text { ses parents pour vivre comme } \\
\text { une grande.» (p. 7) } \\
\text { «En chemin, elle avait rencon- } \\
\text { tré une autre tortue qui s'appelait } \\
\text { Pouce. Pouce était, lui aussi, } \\
\text { parti très tôt de chez ses parents } \\
\text { pour vivre comme un grand } \\
\text { [...] ce fut très facile, parce } \\
\text { qu'ils avaient les mêmes idées } \\
\text { et qu'ils étaient d'accord sur } \\
\text { tout.» (p. 7) } \\
\text { «Pochée était tellement triste } \\
\text { qu'elle souhaita être morte.» } \\
\text { (p. 10) } \\
\text { («il plut», «la rivière grossit», } \\
\text { «la piscine avait débordé», } \\
\text { «cabane mouillée» p. 10) }\end{array}$ \\
\hline
\end{tabular}

Figure 1. - Les traces d'engagement du scripteur dans une production proche du texte source (scripteur fort - 339 mots).

* Les extraits de textes cités ici et dans la suite de l'article n'ont subi aucune correction. 


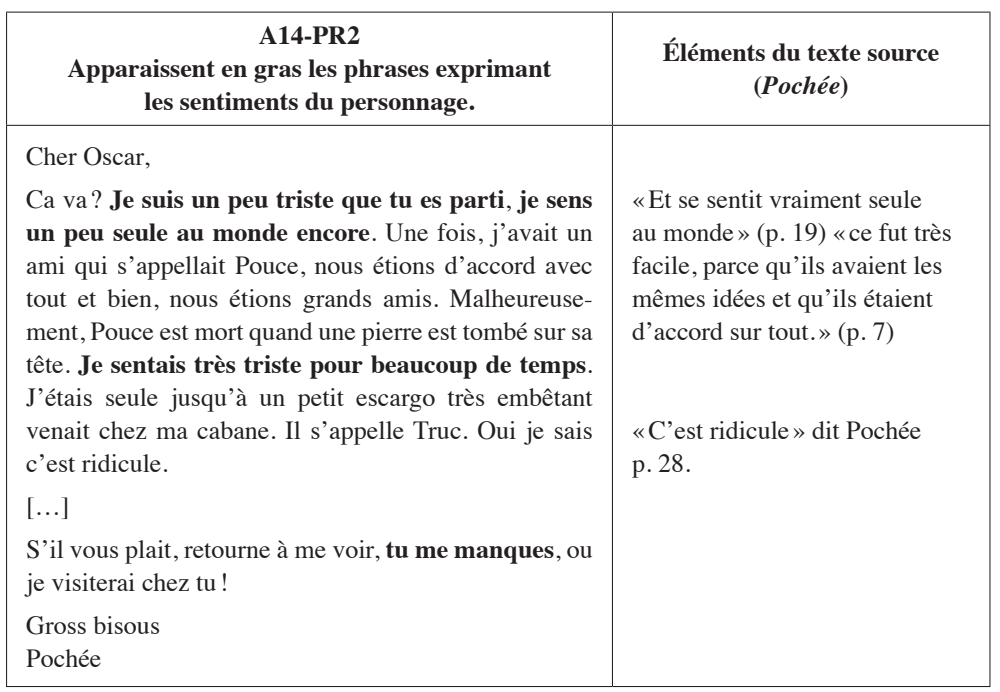

Figure 2. - Les traces d'engagement du scripteur dans une production proche du texte source (scripteur plus faible - 234 mots).

Pour A14, l'effort porté sur l'élaboration au plan linguistique est visible. L'apprenante ne se contente pas de faire un rappel des éléments linguistiques qu'elle a appris, elle se les réapproprie comme le montre l'exemple : «J'ai pensé qu'elle était le plus jolie endroit du monde», alors que le texte dit «je n'ai jamais vu d'endroit aussi joli» (Seyvos, p. 53). Les sentiments sont ici peu développés, et lorsqu'ils le sont, c'est toujours un peu de la même manière, en recourant à l'adjectif «triste»: «Je suis un peu triste que tu es parti», «Je sentais très triste pour beaucoup de temps», «il était un peu triste comme moi je pense». Si la répétition de ce terme met sans doute en avant la lecture de l'apprenante et l'interprétation qu'elle a du texte, on peut sans doute aussi affirmer que cette apprenante est peu outillée sur le plan linguistique pour exprimer des sentiments, même si on lit à la fin de son texte «tu me manques», une expression difficile pour les anglophones.

On peut dire que l'engagement du lecteur-scripteur dans ce type de production se situe essentiellement dans le travail de mémorisation et de remémoration du vocabulaire. Les écrits de ce type sont donc marqués par une forte impression de «collage» ainsi que par un tissage plus ou moins élaboré des éléments entre eux. Ce tissage aboutit toutefois à chaque fois à une production pertinente, conforme aux exigences et faisant sens. 


\subsubsection{Traces d'engagement relevées dans des productions avec apports personnels plus nombreux}

Nous présenterons ici quelques exemples de productions qui s'éloignent de manière plus prononcée du livre, où la restitution du texte source peut être moins précise (que ce soit du point de vue des idées ou du point de vue linguistique) que dans les productions précédemment analysées. Cela étant, qu'il s'opère au plan linguistique ou sémantique, le tissage peut y être, de par son originalité, sa finesse ou son audace, particulièrement remarquable.

\subsubsection{Appropriation linguistique, tissage et hybridité}

Lorsqu'A7 écrit : «Il est parti très heureusement pour aller voir son famille, danser et sauter dans les flaques et aller voir ses amis», il ne se contente pas de reprendre le texte ${ }^{12}$ mais le reformule à sa façon. Ainsi, il «joue» avec le texte source et crée une nouvelle image, pleine de pertinence.

\subsubsection{La lettre, type d'écrit fécond}

Dans le livre, les lettres écrites par Pochée et adressées à elle-même constituent une illustration du fait que celui qui écrit une lettre «est à

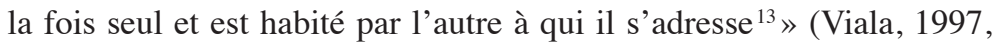
p. 433). Nous trouvons de nombreux exemples de cette adresse au destinataire dans les productions analysées. En voici deux :

A15 : «Il m'a demandé pour t'aider, parce qu'il avait besoin de l'eau. Le jour était très chaud, tu vois, et ses petites cornes étaient sèches.» Un peu plus loin : «Il s'appelle Truc apparemment. C'est très ridicule, non?!» On remarque ici un ajout de la part de l'apprenant : la modalisation, ce qui n'est pas dénué d'humour. Enfin plus loin, A15

12. «Il nageait dans les petites flaques de couleur pour les mélanger puis se promenait lentement sur la feuille, dans tous les sens.» (Seyvos, p. 35) Épisode de Truc l'escargot en train de peindre.

13. «Celui qui écrit une lettre est à la fois seul et habité par celui à qui il s'adresse. Seul, car, à la différence du dialogue oral ou de l'exercice oratoire en public, il ne peut ajuster son texte en cours d'énonciation selon les attitudes et les réactions du destinataire. Et "est habité par l'autre" parce qu'il doit, en conséquence, imaginer les réactions possibles, anticiper sur les effets.» (Viala, 1997, p. 433) 
écrit : «Je lui ai dit de ma vie comme je te dit» [je lui ai raconté ma vie comme je le fais avec toi maintenant]. Cette adresse au destinataire est la marque d'une forte présence du scripteur dans son texte.

A5: «Comme tu es très sympathique, et un peu comme moi, je sais que tu aimeras recevoir cette lettre. Je veux te raconter un peu plus de ma vie, et je sais que tu me comprendras.»

\subsubsection{Un exemple de production remarquable, celle d'A19 (480 mots)} A19-PR2 :

Cher Oscar,

J'étais triste que je ne pouvais pas finir mon histoire parce que tes parents sont arrives. Donc, j'ai décidé d'écrire à toi pour finir ma histoire.

Tu te souviens que je t'ai raconté à propos de la mort de Pouce? Deux semaines plus tard, j'ai eu un invité, un petit escargot, un peu desséché. Il s'a appelé Truc. Il m'a demande si il pourait entre parce qu'il mourait. Il avait besoin de l'eau. Je voulais d'être seul, mais il n'a pas comprendré. Finalement, je l'ai laissé entrer.

Quelle erreur! J'étais tellement aggacé ! Il a voulu utiliser tous mes affaires et les affaires de Pouce! Par exemple, il a essayé de s'asseoir sur le coussin de Pouce! Il étais chez moi seulement cinq minutes. Je ne l'ai pas crue ! De plus, il a voulu manger la nourriture que Pouce et moi ont collecté. Je l'ai dit qu'il pouvait manger la laitue au fond du jardin. J'étais aussi exaspéré parce qu'il a ronflé quand il a dormi ! [...]

L'engagement du lecteur-scripteur se reflète dans cette production non seulement par sa longueur remarquable (480 mots), mais aussi par la présence d'un préambule et d'une partie conclusive, où l'attention envers le destinataire et la présence de ce dernier dans le texte sont particulièrement marquées. Pour autant, il s'agit d'une copie de niveau 2-3. On note ici également la place importante de l'affectif et de l'émotionnel. Dès le préambule, est exprimé le regret de Pochée de n'avoir pu poursuivre son récit à cause de l'arrivée des parents d'Oscar. Ce qui diffère ici de certains autres écrits, c'est que les sentiments ne sont pas seulement exprimés avec des expressions marquant le recul comme «j'étais aussi exaspéré parce qu'il a ronflé quand il a dormi», mais qu'ils s'inscrivent aussi dans le récit sous forme d'exclamatives, ce qui rend le récit particulièrement vivant. À titre d'exemples, nous trouvons «Finalement, je l'ai laissé entrer. Quelle erreur!», «Je ne l'ai pas crue!». Dans cette production, on remarque qu'il y a très peu de phrases reprises textuellement : il s'agit plutôt d'un tissage, d'un 
rebrassage de choses dites depuis la perspective de Pochée. Ainsi des associations sont faites qui n'apparaissent pas dans le livre: «J'ai mis tous mes affaires dans une valise et $\mathrm{j}$ 'ai dit au revoir à ma maison.» Si l'on peut considérer qu'il s'agit simplement d'une manière de résumer le texte de Florence Seyvos, on peut aussi voir dans le choix de l'expression «dire au revoir à sa maison », une personnification qui n'est pas sans évoquer, pour nous, ce que Lacelle et Langlade (2007) appellent «la concrétisation imageante et auditive», phénomène qui consiste, de la part de l'apprenant, en la production d'images et de sons en complément de l'œuvre.

On note également dans cette production que l'étudiant établit des liens de causalité entre les évènements et les actions des personnages (ce que Lacelle et Langlade [2007] appellent «la cohérence mimétique») et qu'il [re]scénarise des éléments de l'intrigue à partir de son propre imaginaire (ce que Lacelle et Langlade [2007] appellent «l'activité fantasmatique»). Qu'il s'agisse d'ajouts au livre tels que nous les avons mentionnés plus haut ou de re-scénarisation des éléments de l'intrigue, ces deux cas de figure n'indiquent pas, bien au contraire, qu'il n'y a pas eu engagement au niveau de la lecture puis de l'écriture. L'apprenant, d'une certaine manière, a rempli les blancs du texte et a réinterprété celui-ci à sa façon.

Parmi les autres éléments présents dans cette production, on note aussi des marques d'ancrage dans le temps et l'espace, la présence du style indirect, marque d'une prise de risque plus importante du point de vue linguistique. On note encore la présence de phrases complexes du type «malgré le fait qu'il m'énerve, il savait peindre et désherber» (phrase présente dans la suite de la production mais non reproduite ici). On remarque aussi la mémorisation par le scripteur anglophone d'expressions qui sont généralement source de problèmes, comme dans la phrase suivante «Pouce me manquait» (phrase non reproduite dans l'extrait présenté).

En ce qui concerne le «texte du lecteur», il est évident qu'il apparait ici de manière incontestable et avec beaucoup de finesse. Pouce, le personnage disparu au début du livre n'est pas absent du texte et des pensées de Pochée, mais continue, comme c'est le cas dans l'épilogue du livre, à être vivant et présent dans les pensées de Pochée. Le texte ne manque pas de nous rappeler l'importance de Pouce pour Pochée : «Comme tu sais, je pense à Pouce tout le temps [...]», y compris longtemps après sa disparition : «Le petit escargot partait [...] j'ai voulu voir Pouce»; «Pouce me manquait. Il y avais beaucoup de choses dans 
ma maison que me rappelle de Pouce»; «J'ai rencontré un hérisson $[\ldots]$ nous nous sommes entendus très bien, un peu comme Pouce et moi.»

\subsubsection{Influence du style du texte sur l'écriture des apprenants- scripteurs}

Nières-Chevrel (2009) parle de «l'écriture minimaliste dans Pochée» (p. 85). Il nous semble que dans certains cas l'apprenant-scripteur a été influencé par le style du livre et produit un texte qu'on pourrait également qualifier de «sobre». On pourrait considérer la manière dont s'exprime A15 comme étant son style propre, sa manière d'écrire. On pourrait encore penser que ses compétences scripturales qui ne sont pas celles d'un locuteur natif et qui comportent des insuffisances le poussent à la retenue. On peut cependant aussi penser qu'il a été sensible au style de l'auteur et qu'il n'est pas impossible que l'écriture sous contrainte, dans un genre tel que la greffe, soit le lieu d'une imitation volontaire ou non du style de l'auteur. Considérons par exemple l'extrait suivant :

Après là, je suis parti en voyage très long, et j'ai recontré un hérisson qui s'appelle Pépin. La mère de Pépin, un jour, l'a dit à prendre ses neuf sœurs et ses sept frères pour une promenade et qu'il ne doit les perdre. Mais ses frères et sœurs l'ont perdu ! Pauvre Pépin.

Pépin m'a invité à sa maison et il m'a donné des tartes de myrtle qu'il a cuit. Ils étaient délicieuses! Je lui ai dit ma vie comme je te dit! Le jour prochain j'ai dit «Au revoir» à Pépin et j'ai recommencé mon chemin.

\subsubsection{Influence de l'image sur l'écriture}

Nous avons dans la production d'A7 l'exemple d'un apprenantscripteur qui, à un moment donné, s'inspire de l'image vue plutôt que du texte lu. A7 écrit : «Un jour, j'ai vu de loin un petit hérisson.» Or, si nous regardons le texte source, le texte dit: «Sous un frêne, elle aperçut un hérisson.» Dans le texte de Seyvos, il n'est donc pas question de distance. En revanche, comme nous pouvons le voir sur la reproduction (fig. 3), l'image figure la distance. En arrière-plan, bien que sous un pan de lumière, Pochée apparait à distance de Pépin, représenté au premier plan de l'image, à droite. 


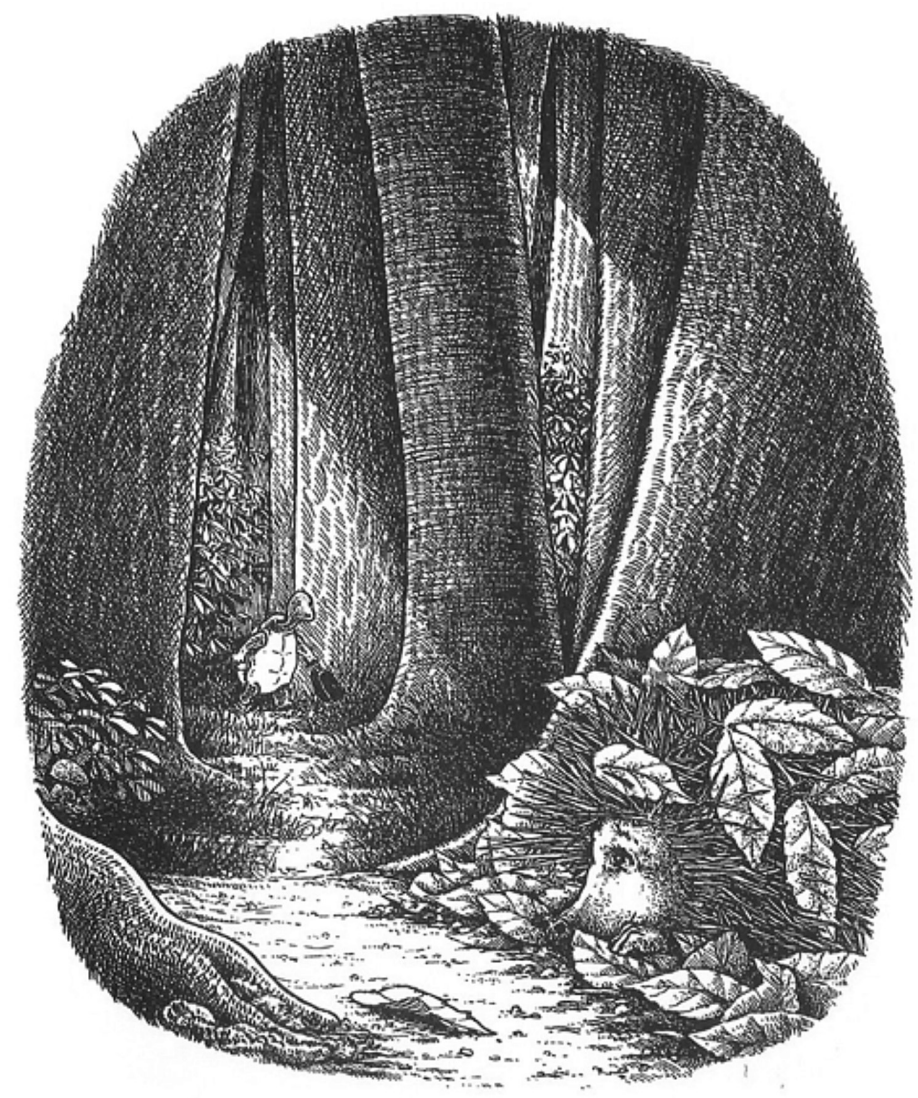

Figure 3. - Image source : Pochée, p. 49.

4.2.3.6. Expression de la subjectivité lectrice dans la production de l'apprenant-scripteur

Un exemple de subjectivité lectrice est la tristesse mise en avant par A11 :

Mais une jour, Pouce avait un accident, et Pouce me partait pour toujours. Ces-jours-là étaient très triste parce que j'étais seulement. Je ressentais solitaire. J'ai décidé écrire des lettres à moi de Pouce. Ces lettres étaient des lettres que Pouce m'écrirait si Pouce n'était pas mort.

D'autres productions mettent en avant la dimension philosophique du livre. Autre exemple de ce type de production, celle d'A7 met en avant la notion de liberté. Dans la production d'A4, en revanche, c'est 
la notion d'amitié qui est centrale. A26, quant à lui, développe la notion de bonheur. Pour lui, le processus de deuil a été accompli.

\subsubsection{Humour et infidélité(s) au texte source}

Dans le corpus de productions analysées, cinq productions comportaient au minimum un clin d'œil humoristique au texte de Seyvos. Nous avons interprété cela comme une marque d'engagement. Bien que très faible linguistiquement, l'une de ces productions faisait de multiples références humoristiques au livre et témoignait ainsi de réelles qualités littéraires. Ce cas de figure et les questions qu'il pose pour l'évaluation ont été soulignés dans la recherche au sujet l'écriture d'invention (Huyn, 2004).

Une question est revenue au fil de nos analyses : devait-on considérer une infidélité au texte source comme une marque de non-engagement du lecteur-scripteur? De notre point de vue, on ne peut pas, en FLE, ne pas valoriser une élaboration personnelle qui montre d'abord la capacité du scripteur à exprimer ses idées en langue cible. De plus, lorsque l'apprenant de langue étrangère s'éloigne du texte source en ajoutant des éléments qui n'y figurent pas mais sont en cohérence avec celui-ci, il fait preuve d'engagement dans la tâche demandée.

\section{Conclusion}

Les résultats de l'analyse mettent à jour un foisonnement de traces d'engagement et révèlent la pertinence d'exercices d'écriture ambitieux à des niveaux peu élevés de maitrise de la langue cible. L'analyse des productions réalisées montre que celles-ci correspondent à des descripteurs du CECR de niveau plus élevé (par exemple, au niveau C2: «peut écrire des histoires simples ou des récits captivants, de manière limpide et fluide et dans un style approprié au genre adopté ${ }^{14} \gg$ ) que ceux auxquels on rattache généralement les productions de ces apprenants.

Les travaux réalisés ont eu lieu peu de temps après la lecture et le travail effectué en classe, et il serait sans doute pertinent d'observer les traces d'appropriation sur le long terme, en proposant un exercice d'écriture créative plus distancié de la phase de lecture-apprentissage. En outre, l'échantillon présenté était relativement restreint (26 apprenants) et, pour cette raison, il n'est pas possible de généraliser nos observations. Cependant, notre recherche présente des résultats qu'il serait

14. CECR, Écriture créative (sous-échelle de la production écrite), p. 52. 
important de confronter à ceux de recherches effectuées auprès d'autres groupes témoins.

Conduite à partir d'un cadre élaboré spécifiquement et transférable, l'analyse nous a permis de conclure que l'ouvrage choisi, bien qu'appartenant au champ de la littérature de jeunesse, a suscité chez des apprenants de niveau universitaire des écrits riches et pertinents où s'est exprimée la subjectivité lectrice.

Les traces d'engagement relevées montrent donc la pertinence de proposer certains textes destinés à la jeunesse à de jeunes adultes, ainsi que des activités d'écriture sous contrainte, d'appropriation et de détournements de textes. Nous pouvons aussi affirmer que l'objectif didactique que nous nous étions fixé initialement est effectivement atteint, et conclure, comme le fait Houdart-Mérot (2008) dans le champ du français langue maternelle, que l'écriture sous contrainte a permis la perception de la dimension oblique de la littérature et la libération de la lecture et de l'écriture.

\section{RÉFÉRENCES BIBLIOGRAPHIQUES}

BARRÉ-De MinIAC, Christine. (2000). Le rapport à l'écriture : aspects théoriques et didactiques. Villeneuve d'Ascq: Presses universitaires du Septentrion.

BaRré-De Miniac, Christine. (2002). Du rapport à l'écriture de l'élève à celui de l'enseignant. Où l'élève interroge l'enseignant. Éduquer, 2. Disponible en ligne sur $<\mathrm{http}: / /$ rechercheseducations.revues.org/283>.

CONSEIL DE L'EuROPE. (2001). Cadre européen commun de référence pour les langues : apprendre, enseigner, évaluer (CECR). Paris : Éditions Didier.

CuQ, Jean-Pierre. (2003). Dictionnaire de didactique du français langue étrangère et seconde. Paris : CLE international.

CuQ, Jean-Pierre et Gruca, Isabelle. (2005). Cours de didactique du français langue étrangère et seconde. Grenoble: Presses universitaires de Grenoble.

Daunay, Bertrand. (2004). Traces d'apprentissage : que reste-t-il d'une séquence didactique? Recherches, 41, 141-170.

Demougin, Patrick. (1998). Littérature et pratique d'écriture en FLE : clarifier les enjeux. Travaux de didactique du FLE, 39, 69-77.

FERRIER, Bertrand. (2006). «Mais tout n'est pas littérature!» Le concept de littérarité appliqué aux romans contemporains pour la jeunesse (19952005) (Thèse de doctorat). Paris, Université Paris-Sorbonne. 
HoudART-MÉrot, Violaine. (2008). Atelier de réécriture et critique littéraire en acte à l'université. Recherches et travaux, 73, 125-137.

HuYn, Jeanne-Antide. (2004). L'écriture d'invention, une pratique innovante. Recherches, 40, 45-59.

LACELLE, Nathalie \& LANGLADE, Gérard. (2007). Former des lecteurs/spectateurs par la lecture subjective des œuvres. Dans J.-L. Dufays (dir.), Enseigner et apprendre la littérature aujourd'hui, pour quoi faire? (p. 56-65). Louvain, Belgique : Presses universitaires de Louvain.

LE Goff, François. (2008). Réflexions sur la réécriture en écriture d'invention. Recherches \& Travaux, 73, 19-34.

Maizonniaux, Christelle. (2013). Les apports de la littérature de jeunesse pour la didactique du FLE en contexte universitaire australien (Thèse de doctorat non publiée). The Australian National University, Canberra (Australie) \& Université Stendhal-Grenoble 3, Grenoble.

Mazauric, Catherine, Fourtanier, Marie-José \& Langlade, Gérard. (2011). Le texte du lecteur. Bruxelles, Belgique : Peter Lang.

NiÈres-Chevrel, Isabelle. (2008). Doubles images, doubles lecteurs : l'intericonicité dans Le Tunnel d'Anthony Browne. Dans C. Pintado, F. Gaïotti \& B. Poulou (dir.), L'album contemporain pour la jeunesse : nouvelles formes, nouveaux lecteurs? (p. 89-99). Bordeaux : Presses universitaires de Bordeaux.

NiÈres-CheVrel, Isabelle. (2009). Introduction à la littérature de jeunesse. Paris : Didier jeunesse.

Rodier, Christian, Bara, Stéphanie \& Bonvallet, Anne-Marguerite. (2011). Écritures créatives. Grenoble : Presses universitaires de Grenoble.

Rouxel, Annie \& LANGLAde, Gérard (dir.). (2004). Le sujet lecteur : lecture subjective et enseignement de la littérature. Rennes : Presses universitaires de Rennes.

Seyvos, Florence. (1994). Pochée. Paris : L’École des loisirs.

Viala, Alain. (1997). Littérature épistolaire. Dans Dictionnaire des genres et notions littéraires (p. 432-436). Paris : Encyclopædia Universalis et Albin Michel. 